\title{
Using Extra Virgin Olive Oil (Evoo) as a Therapy in Angular Cheilitis: Current Scientific Evidence is Rediscovering Our Grandparents' Ancient Tradition
}

\author{
${ }^{1}$ RDH, Freelancer in Canosa di Puglia, Italy \\ ${ }^{2} D D S$, Private Dental Practice, Barletta, Italy \\ ${ }^{3} D D S$, Private Dental Practice, Barletta, Italy \\ ${ }^{4} \mathrm{RDH}$, Freelancer in Barletta, Italy
}

Antonia Sinesi ${ }^{1 *}$, Giovanni Di Leo ${ }^{2}$, Savino Cefola ${ }^{3}$, Ruggiero Damato $^{4}$, Cinzia Casu $^{5}$

Received: March 10, 2021; Accepted: March 21, 2021; Published: March 29, 2021

“Corresponding author: Antonia Sinesi, RDH, Freelancer in Canosa di Puglia, Italy; E-mail: antonia.sinesi@gmail.com

\begin{abstract}
Extra Virgin Olive Oil (EVOO) is an essential food in the Mediterranean diet. Since the last century, it was customary for people in southern Italy to use it to treat various diseases: burns, trauma, cheilitis and inflammatory processes of various kinds. Over time, this practice, the result of ancient knowledge handed down from generation by generation, gradually disappeared and was almost forgotten.

In Southern Italy, precisely all through the countryside of the north of Bari in Apulia, is produced a particular type of EVOO from "Coratina" cultivar characterized by the presence of a high content of polyphenols and other biocoumponds, the highest compared to other cultivars.

Although numerous studies on EVOO have shown its remarkable effectiveness in wound healing, no scientific studies have been proposed for the treatment of Angular Cheilitis(AC).

In this case report, we propose the use of EVOO as a therapeutic aid in the treatment of $\mathrm{AC}$ not responsive to ointments of various kinds: cortisone, antibacterials or antifungals.
\end{abstract}

\section{Introduction}

Angular Cheilitis(AC) is a descriptive diagnosis for an inflammatory skin process of varied etiology occurring at the labial commissure - the angle of the mouth. "Angular", or commissural, refers to a localized lip inflammation (i.e., "cheilitis," from the Greek word "cheilos" meaning "lip") that is distinguishable from the more generalized cheilitides that have different causes [1].

The disease may appear as an isolated condition or as part of certain systemic diseases/conditions (such as avitaminosis, iron deficiency, celiac disease [2] or local infections (e.g., herpes, oral candidiasis, Staphylococcus aureus infection [2]. Cheilitis can also be a symptom of a contact reaction to an irritant or allergen, or may be caused by sun exposure (actinic cheilitis) or drug intake, especially retinoids. Older age is a predisposing factor when associated with tissue collapse in the corner of the mouth due to loss of teeth and decreased vertical dimension of occlusion as a result of missing or inadequate dentures. Generally, the forms most reported in the literature are angular, contact (allergic and irritant), actinic, glandular, granulomatous, exfoliative and plasma cell cheilitis[3].

The literature describes several cases in which Extra Virgin Olive Oil (EVOO) is used, such as in cases of mucositis following chemotherapy [4], xerostomia and burning mouth syndrome $[5,6]$, in the prevention of initial enamel erosion [7], in the treatment of major traumatic ulcers [8].

There are no studies reported in the literature where EVOO has been used for the treatment of AC.

\section{Methods and Materials}

A 65-year-old male patient presented to our dental office for an oral hygiene session. During the inspection we noticed a notable bilateral AC (Figures 1, 2). The patient reported that the lesion had been present for about two months and that, being a general practitioner, he had treated it with ointments of various kinds: cortisone, antibacterials, antifungals, without ever achieving a definitive cure. He also reported times when the lesion faded and times when it exacerbated. From the compilation of the medical history only a previous angioplasty emerged. The oral hygiene session was not performed in order to prevent the lesion from bleeding. We dismissed the patient prescribing a therapy based on EVOO, "Coratina" cultivar. We advised the patient to apply the oil to the lesions 4 times a day. We also recommended him to do it with a light circular massage, making sure that the oil was absorbed both externally and internally to the lesion, and being careful not to perform any movements with the mouth that could cause the breakage of a possible crust present on the lesions. We therefore advised him to carry out the first dressing in the 


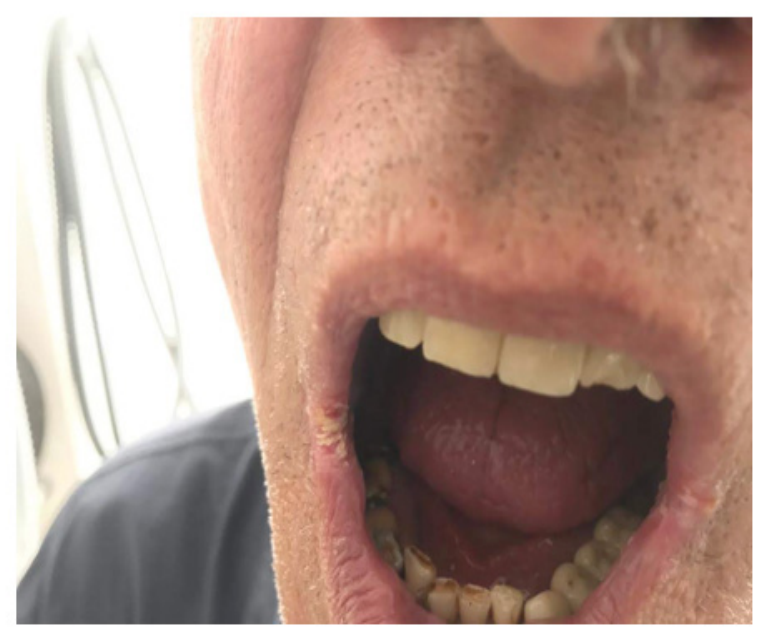

Figure 1: Initial state: Angular Cheilitis

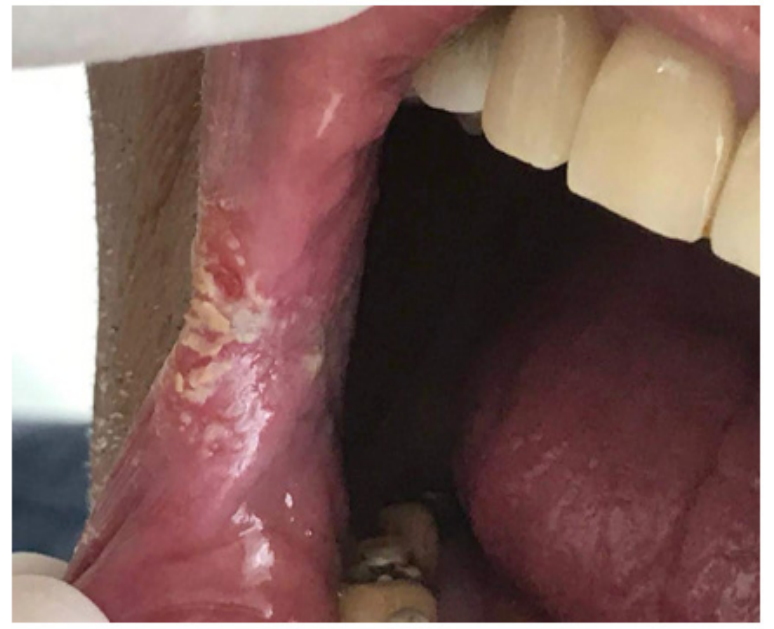

Figure 2: Initial state: Angular Cheilitis

morning as soon as he woke up before he started to speak.

\section{Results}

After 7 days from the first visit, we revisited the patient who presented a better looking and healing lesion (Figures3,4). We recommended repeating the therapy for another 10 days. At the next checkup, the bilateral cheilitis was healed (Figure5).

\section{Discussion}

Clinically, $\mathrm{AC}$ is a frequent condition characterized by erythema rhagades, ulcerations, and crusting of the lip corners and the adjacent skin, either in one commissure or both. This form is downright different from other well-known possibly dysplastic labial diseases [9].

The angles of the mouth are points of interface for squamous epithelium of the face and oral mucosa. They are also a mechanically dynamic hinge for the oral aperture that endures more motion and tensile forces than the rest of the lips. Thus, the commissures are especially susceptible to certain stresses [10].

Most cases of AC are ultimately due to physical maceration at the angular commissures due to overexposure to saliva. The digestive enzymes in saliva can act even on oral tissues following prolonged contact. Continued saliva exposure induces a contact dermatitis and eczematous reaction at the commissures. The compromised integrity of the stratum corneum epithelium allows local commensal organisms to infect the area. Frequently, colonizing Candida albicans establishes and invades the susceptible tissue. This may then allow bacterial superinfection with staph and strep species. Thus, risk factors are those that increase saliva retention at the commissures and exposure to culprit microbes, cause direct tissue inflammation, or inhibit wound healing and immunity [3]. 


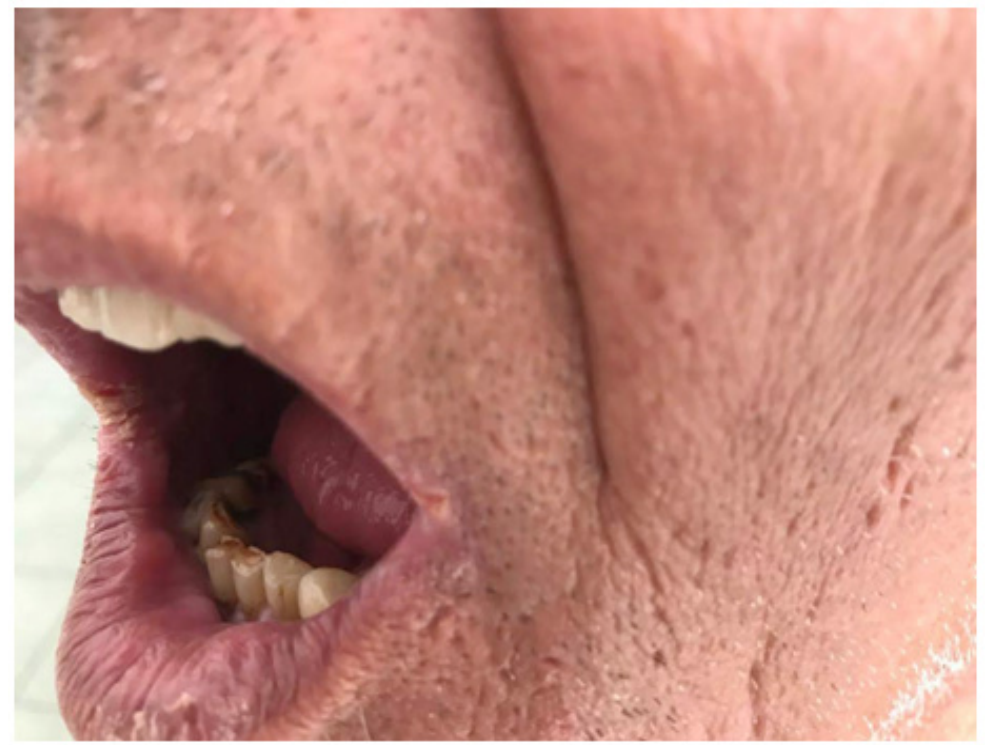

Figure 3: Seven days after EVOO application

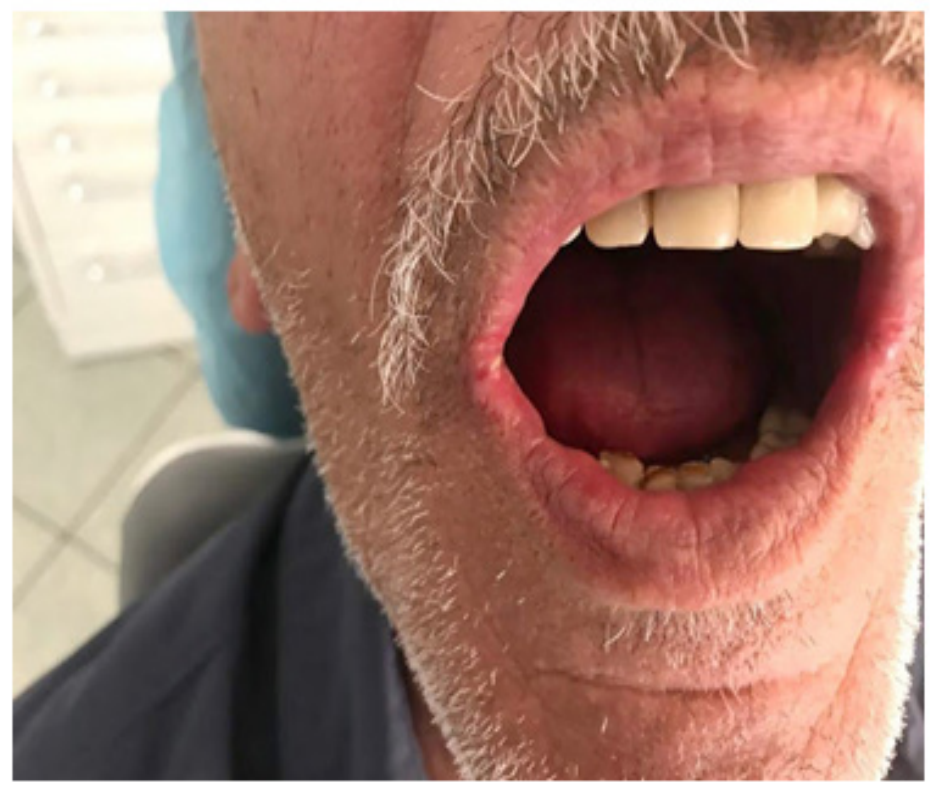

Figure 4: Seven days after EVOO application

The most comprehensive categorization of AC clinical spectrum was formulated in 1986 by Ohman and co-workers. Small rhagades limited to the corner lips with slight skin involvement were defined as type I; deeper and more extensive lesions with uneven borders were described as type II; several rhagades extending from the lip corners into the perioral skin were referred as type III. Finally, a diffuse erythema spread in the skin surrounding the vermillion border would fall into type IV [10].To date, this classification is still considered reliable and exhaustive as the most recent case series show [11].
Conventional therapy for AC involves the use of antifungal, antiseptics or antibiotics drugs, topical glucocorticoids, topical barriers (vaseline, emollients or lip balm) to prevent tissue maceration if the cause is excessive saliva. Photodynamic therapy [12] and L.L.L.T. (low level laser therapy) [13] have been shown to be helpful for non-pharmacological therapy in AC.

According to a literature review carried out in 2019 by a team of Italian researchers regarding the various treatments for AC experimented over the years and documented in scientific articles, despite antifungals being the first-line treatment for 


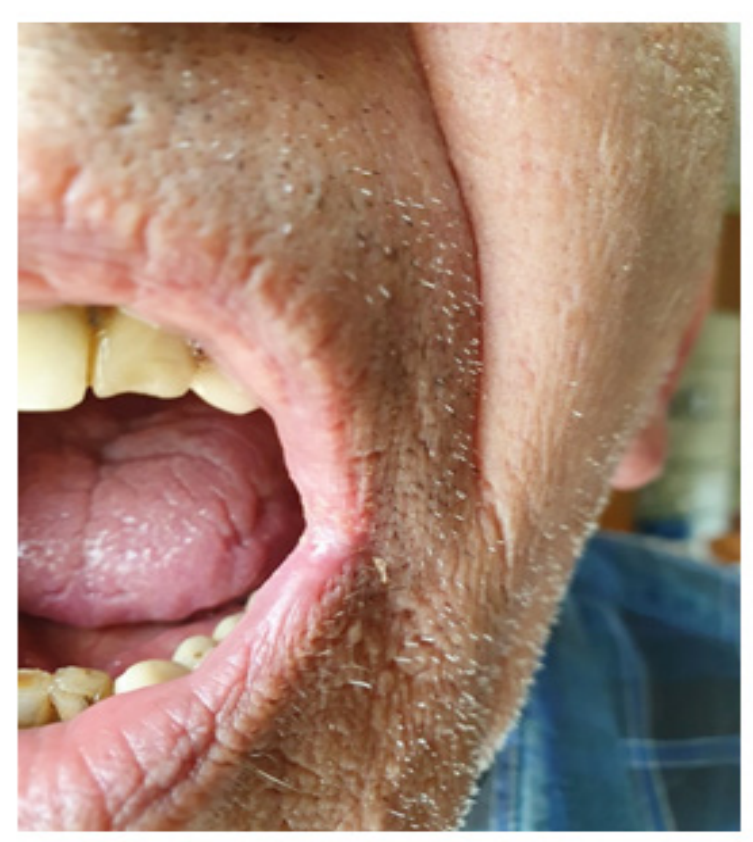

\section{Figure 5: Seventeen days after EVOO application}

most of clinicians, very limited scientific evidence supports their reliability, with just two RCTs published between the 70 's and the 80's. Furthermore, alternative topical treatments, various techniques of occlusal vertical dimension restoration, B-vitamin supplementation, anti-drooling prosthetic device, and photodynamic therapy have been experimented and proposed, mostly in the form of case reports or case series on a small number of individuals [14].

In the review the use of ozonized olive oil emerges among the topical treatments [1].For this reason, and aware of its extraordinary health properties [15], we decided to use olive oil from the "Coratina" cultivar as a topical dressing for the resolution of a case of AC.

The "Coratina" cultivar is grown in a large area in the north of Bari, Apulia. It produces a particular type of oil with a high polyphenol content. The extraordinary antioxidant properties of the EVOO from "Coratina" cultivar are related to the presence and concentration of tocopherols, carotenoids and phenolic compounds that are of great importance to human health [16].

EVOO phenolic compounds have also been widely recognized as anti-inflammatory agents due to their ability to modulate the inappropriate activation of NF-kB signaling, thereby limiting its deleterious effects in all tissues. Indeed, their ability to modulate NF-kB is closely linked to their antioxidant nature. EVOO polyphenols are known to be primarily antioxidant in the broadest sense of the term, being able to directly eliminate oxidant species and increase endogenous cellular antioxidant defences [17].

Plant polyphenols are produced by plants to defend themselves against pests such as bacteria, fungi and insects, to which they are toxic. The main EVOO polyphenols, hydroxytyrosol (2-(3,4-dihydroxyphenyl) ethanol, HT) and tyrosol (2-(4-hydroxyphenyl) ethanol, Tyr), and other less concentrated phenolic compounds are biologically important not only for their antioxidant activity [18], but also for their involvement in modulating numerous intracellular signals [19-23] involving other beneficial effects than free radical scavenging. Indeed, EVOO polyphenols are well known for their anti-inflammatory effects, exerted by down-regulating inflammatory mediators via transcriptional or post-transcriptional mechanisms and modulating the activation of kinases involved in the initiation of the inflammatory process at different levels $[19,24]$.

In an in vivo study on mice was described the role of olive oil in healing pressure ulcers. Following daily oral application of coldpressed olive oil for 14 days, an improvement in wound healing was observed. This was achieved through anti-inflammatory and antioxidant effects, and the promotion of dermal reconstruction. Olive oil was found to reduce the production of nitric oxide and reactive oxygen species and thus oxidative damage. Collagen deposition, myofibroblastic differentiation and wound contraction were also accelerated by oral administration of olive oil [25].

In a randomized, double-blind, comparator-controlled, parallelgroup clinical study, 60 patients with chronic wounds were treated twice a day through either the application of a cream containing aloe vera and olive oil (composition not available) or phenytoin cream, as the standard treatment, for a period of 30 days. The wound size, depth, and edges, together with the associated pain, were significantly reduced by both treatments. However, the aloe 
vera and olive oil cream was more effective [26].

The healing effect of cold-pressed olive oil was evaluated in comparison with the lyophilized powder of sea buckthorn leaf aqueous extract and a silver sulfadiazine and saline (control) group on full-thickness burn wounds in rats. Wound contraction was observed for 28 days and occurred faster in groups treated with olive oil, sea buckthorn extract, and a mixture of sea buckthorn extract and olive oil than in the silver sulfadiazine and saline group. Using a mixture of olive oil and sea buckthorn extract, a synergistic effect was observed, which resulted in improved wound-healing characteristics and antibacterial effects [27].

A traditional Iranian olive oil ointment (a mixture of olive oil, sesame oil, and honey) was tested in a randomized controlled clinical study including 30 patients. The control group was treated with a mafenide acetate ointment (8.5\%). The final study result is that the olive oil ointment is a useful treatment for burn wounds, as it prevents infections and accelerates tissue repair. The mean time of granulation tissue formation in the olive oil ointment group was 12 days, whereas it was 17 days for the mafenide acetate group [28].

Karygianni L et. al. states that maslinic acid isolated from leaves of 0 . europae aproved to be highly effective, even in very low concentrations in the range $9.8-25 \mu \mathrm{g} / \mathrm{mL}$, against oral streptococci and anaerobic pathogenic bacteria such as Porphyromonasgingivalis and Fusobacteriumnucleatum. Maslinic acid belongs to natural pentacyclic triterpenoids, which are able to damage the cell envelope of both Gram-positive and Gram-negative bacteri(kurek 2012). The other four compounds isolated from 0. europaea, namely oleuropein, oleocanthal, hydroxytyrosol and oleacein showed more moderate inhibitory effects than maslinic acid against the Gram-negative anaerobic P. gingivalis and F. nucleatum [29].4-Hydroxytyrosol also exerted bactericidal activity against the gram-positive $\mathrm{S}$. aureus and the virulent staphylococcal enterotoxin A [30]. Oleocanthal and oleacein are two aldehydic compounds in olive oil with great structural similarity [31]. To date, both substances have been shown to be natural non-steroidal, antioxidant and antiinflammatory compounds [32,33] Scotece M. et al. clarified the active anti-inflammatory role of oleocanthal [34]. In particular, oleocanthal interferes with the activity of lipopolysaccharidestimulated macrophages and chondrocytes by inhibiting nitric oxide (NO), interleukin (IL-6, IL-1 $\beta$ ) and tumour necrosis factor $\alpha$ (TNF- $\alpha$ ) production [34].

In a study by Bisignano C. et al. it was shown that a metabolite of oleuropein was effective against S.epidermidis and aureus and could therefore be a potential source of natural antimicrobials for the treatment of skin infections [35].A study by Diallinas G. et al. investigated the antifungal activity of a series of chemically synthesised Hydroxytyrosol (HT) analogues. HT is one of the main phenolic compounds in olive oil and has been shown to possess antioxidant, antiproliferative, pro-apoptotic and antiinflammatory anti-radical activities. The study shows that antifungal HT analogues have unprecedented efficiency in destroying the fungal plasma membrane [36].

\section{Conclusion}

The use of EVOO in various oral diseases has been being a widespread practice in our region, Apulia, in the south of Italy, already for several centuries, handed down by our grandparents. Therefore, we are not surprised by the healing of AC in a patient refractory to conventional therapies using only and exclusively EVOO. The use of EVOO can be regarded as an alternative therapy to conventional drug therapy.

\section{References}

1. Briel A, Moellmann C, Mattulat B, Schauer F, Kiritsi D, Schmidt E, et al. Bullous pemphigoid in infants: characteristics, diagnosis and treatment. Orphanet J Rare Dis. 2014;9(1):185.

2. Weston WL, Morelli JG, Huff JC. Misdiagnosis, Treatments, and Outcomes in the Immunobullous Diseases in Children. Pediatr Dermatol. 1997;14(4):264-272.

3. Zinman O, Amitai D, Cohen, Arnon D, Feinmesser M, Mimouni D, et al. Bullous pemphigoid in infancy: Clinical and epidemiologic characteristics. J Am Acad Dermatol. 2008;58(1):41-48.

4. Belzile E, Funaro D, Powell J. Localized vulvar bullous pemphigoid of childhood: A rare cause of persistent vulvar erosions in children. Pediatr Dermatol. 2019;36(3):349-351.

5. Voltan E, Maeda JY, Silva MAM, Maruta CW, Santi CG, Zimbres S, et al. Childhood Bullous Pemphigoid: Report of Three Cases. J Dermatol. 2005;32(5):387-392.

6. Petronius D, Bergman R. Bullous Pemphigoid in Two Young Infants. Pediatr Dermatol. 2002;19(2):119-121.

7. De Pablo MI, Enseñat MA, Vicente A, Gilaberte M, Mascaró JM. Childhood bullous pemphigoid: clinical and immunological findings in a series of 4 cases. Arch Dermatol. 2007;143(2):215-220.

8. Erbagci Z. Childhood Bullous Pemphigoid Following Hepatitis B Immunization. J Dermatol. 2002;29(12):781-785.

9. Wu KG, Chou CS, Hsu CL, Lee ML, Chen CJ, et al. Childhood Bullous Pemphigoid: A Case Report and Literature Review. J Clin Exp Dermatol Res. 2013;S6:010. doi:10.4172/2155-9554.S6-010

10. Xiao T, Li B, Wang Y, He C, Chen H. Childhood bullous pemphigoid treated by i.v. immunoglobulin. J Dermatol. 2007;34(9):650-653.

11. Nemeth AJ, Klein AD, Gould EW, Schachner LA. Childhood bullous pemphigoid. Clinical and immunologic features, treatment, and prognosis. Arch Dermatol. 1991;127(3):378-386

12. Calonje Eduardo, Brenn Thomas, Lazar Alexander, Phillip H. McKee's pathology of the skin: with clinical correlations. 4th ed. ed. Elsevier/ Saunders. 2012:1803.

13. Reis-Filho E, Silva TdA, Aguirre, Lima, Reis CMS. Bullous pemphigoid in a 3-month-old infant: case report and literature review of this dermatosis in childhood. An Bras Dermatol. 2013;88(6):961-965.

14. Sáenz AM, González F, Cirocco A, Tacaronte IM, Fajardo JE, Calebotta A. Childhood bullous pemphigoid: a case report and 10-year follow up. 
Int J Dermatol. 2007;46(5):508-510.

15. Motegi S, Abe M, Tamura A, Ishii N, Hashimoto T, Ishikawa O. Childhood Bullous Pemphigoid Successfully Treated with Diaminodiphenyl Sulfone. J Dermatol. 2005;32(10):809-812.
16. Schulze J, Bader P, Henke U, Rose MA, Zielen S. Severe Bullous Pemphigoid in an Infant - Successful Treatment with Rituximab. Pediatr Dermatol. 2008;25(4):462-465. 